\title{
Controllability Analysis of Reactive Magnetron Sputtering Process
}

\author{
Z. $\operatorname{AHMAD}^{a, *}$ AND B. ABDALLAH ${ }^{b}$ \\ Scientific Services $^{a} /$ Physics $^{b}$ Department Atomic Energy Commission of Syria, P.O. Box 6091, Damascus, Syria
}

(Received December 18, 2011; in final form March 11, 2012)

Reactive magnetron sputtering deposition is one of the major established techniques for deposition of both metallic and nonmetallic thin films on various substrates; it is a very nonlinear process, and exhibits hysteresis behavior with respect to the reactive gas flow. This nonlinearity is characterized by a sudden change in sputtering rate and fraction of compound formation. Most of the problems encountered in the preparation of compound films by reactive sputtering are due to the hysteresis effect. The industrial applications request high rate deposition processes. To meet this demand, it is necessary to have a very good control system of such processes and to ensure a stable sputtering in the transition mode by using closed loop reactive gas control. Therefore, the controllability analysis is an important issue. The aim of this paper is to study the controllability of reactive sputtering process, especially in the transition region; a simple mathematical model based on Berg's original proposal is used. Analysis results show that the reactive magnetron sputtering process shows unstable behavior in the transition region and it is a controllable process.

DOI: 10.12693/APhysPolA.123.3

PACS: 02.30.Yy, 68.55.-a

\section{Introduction}

Magnetron sputtering deposition is one of the major established techniques for deposition of both metallic and nonmetallic thin films on various substrates. Reactive sputtering for thin film coatings is used in numerous industrial applications. By simply adding a gas that reacts with the sputtered material, depending on the reactive gas used it is possible to form a wide variety of useful compound thin film coatings, such as oxides, nitrides, carbides which have attractive physical and chemical characteristics such as high strength, hardness, toughness and high corrosion resistance. At first sight, this may seem very simple. However, the reaction mechanisms between the sputtered material and the reactive gas may cause some processing stability problems, and a trade off between combining a high deposition rate and true compound stoichiometry of the deposited film.

Reactive magnetron sputtering process can be divided into three modes: (i) metallic, (ii) transition and (iii) reactive, depending on the amount of reactive gas used [1]. The deposition rate is extremely sensitive to the supply of the reactive gas. A very low supply of the reactive gas will cause high rate metallic sputtering, but may give rise to an understoichiometric composition of the deposited film. A too high supply of the reactive gas will allow for stoichiometric composition of the deposited film, but will cause poisoning of the target surface, which may reduce the deposition rate significantly. This behaviour points out that there may exist optimum processing conditions where both high rate and stoichiometric film composition may be obtained.

In many cases, the main reason for this complication is that as well as forming a compound of the deposited

\footnotetext{
${ }^{*}$ corresponding author; e-mail: pscientific@aec.org.sy
}

film, compound formation will also take place at the surface of the sputtering target (target poisoning). Normally, the sputtering yield of the compound material is substantially lower than the sputtering yield of the elemental target material. This causes the deposition rate to decrease as the supply of the reactive gas increases. The relationship between the film composition and supply of reactive gas is very nonlinear. This is also the case for the deposition rate versus the supply of the reactive gas. Therefore, reactive sputtering processes controlled by the supply of the reactive gas exhibit quite complex processing behaviour $[2,3]$.

The aim of this paper is to explain how different parameters affect the controllability of reactive sputtering process, especially in the transition region; a simple mathematical model based on Berg's original proposal is used. Analysis results show that the reactive magnetron sputtering process exhibits unstable behavior in the transition region and it is a controllable process.

\section{Process control and hysteresis effect}

Figure 1 shows schematically the well known and important feature of reactive sputtering at a constant partial pressure of nonreactive gas. This feature is also known as hysteresis behaviour of reactive magnetron sputtering. Reactive magnetron sputtering process can be divided into three modes: (i) metallic, (ii) transition and (iii) reactive, depending on the amount of reactive gas used [1]. At low values of the reactive gas flow, almost all the available reactive gas is gettered by the sputtered metal (Fig. 1a). As a result, no essential change in reactive gas pressure and target voltage is observed from the background level and the deposited film is metal-rich (metallic mode).

This situation persists until the flow reaches a critical value, where the flow rate of the reactive gas into the chamber becomes higher than the gettering rate of the 


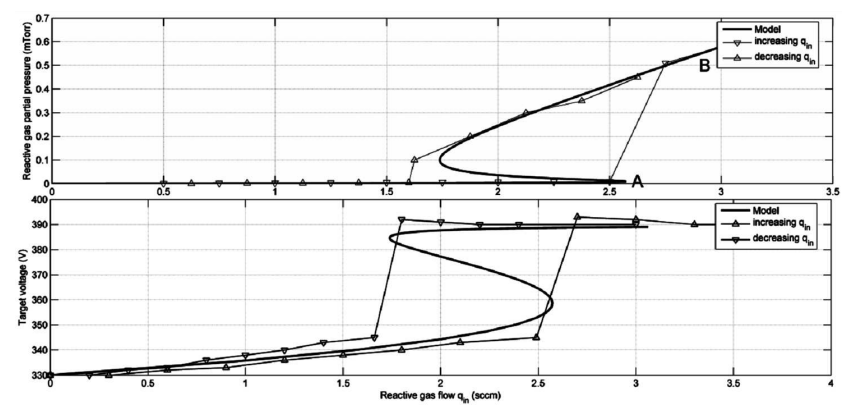

Fig. 1. Hysteresis behaviours of (a) reactive gas pressure and (b) target (cathode) voltage as a function of reactive gas flow rate. Experimental [10] (points).

sputtered metal, which then leads to a sudden increase in the partial pressure of the reactive gas. This dramatic change in the reactive gas pressure is a direct result of the poisoning of the target surface. Consequently, a fewer metal atoms are sputtered and less reactive gas is consumed in the reaction (reactive mode). The deposited film is then gas-rich. Any further increase in the reactive gas flow just leads to a linear increase in its partial pressure. If the flow rate of the reactive gas is reduced following an increase in partial pressure to a high level, partial pressure will not decrease following the same trajectory as it increased and a return to the metallic mode is delayed. This is because reactive gas partial pressure remains high until the compound layer on the surface of sputtered target is fully removed and the metal is again exposed to be sputtered once more. As a result, the consumption of the reactive gas increases and partial pressure decreases to the background level and the deposited film is metal-rich again.

Target voltage changes depending on the target materials when the target becomes fully poisoned due to the increased or decreased secondary electron emission coefficient of the nitrided or carbided or oxided target surface $[4,5]$. For example, in the case of titanium or chromium, the target voltage increases with increasing reactive gas partial pressure. In contrast, the target voltage decreases sharply when aluminum or silicon is used as a target material.

An example for the voltage increase case is given in Fig. 1b. As it can be seen in this figure, the target voltage initially increases as the reactive gas flow is increased, but there is a sharp increase in the target voltage which is the point during which the target becomes poisoned. Once the target is poisoned, the target voltage remains high even when the flow is reduced. It is not until the compound layer on the target surface is broken through that the cathode voltage returns the lower values corresponding to the metallic deposition mode. A closed hysteresis loop is formed in this way. However, the sputtering rates always decrease with increasing reactive gas partial pressure.
It is sometimes very beneficial to operate the process inside the hysteresis region (transition mode). To be able to do this, however, fast and sophisticated process control systems are required. The optimum design of such a control system depends on the dynamic behaviour of the process to be controlled. This dynamic is in turn affected by a number of different parameters such as target material, reactive gas used, pumping speed or chamber volume. Models describing the dynamic behaviour of the reactive sputtering have been described elsewhere $[6,7]$. Several control methods of process stabilization and methods of control to prevent instability have been summarized in Refs. [2] and [3]. Plasma emission monitoring (PEM) and target voltage control techniques have proved to be very reliable in controlling reactive sputtering processes. Especially, target voltage control technique is a simple and inexpensive method. However, the target voltage control can only be used under the conditions where the voltage difference between the metallic target material and the reaction product is sufficiently large [2].

\section{Outline of Berg's model}

\subsection{Rate equations for compound fractions and pressure}

Assuming the ideal gas law and uniform pressure in the chamber, the rate change of the reactive gas (partial) pressure can be expressed as

$$
\frac{\mathrm{d} p}{\mathrm{~d} t}=\frac{k_{\mathrm{B}} T}{V}\left(q_{\mathrm{in}}-q_{\mathrm{t}}-q_{\mathrm{s}}-q_{\mathrm{pump}}\right),
$$

where $k_{\mathrm{B}}$ is the Boltzmann constant, $T$ is temperature and $V$ is the volume of chamber. $q_{\text {in }}$ is the inflow rate of reactive gas, $q_{\mathrm{t}}$ and $q_{\mathrm{s}}$ are, respectively, the consumption rates at the target and substrate surfaces, and $q_{\text {pump }}$ is the outflow rate by the vacuum pump. Units for these quantities are all in (number of molecules/time). Notice that the gettering effect of the chamber walls is neglected here because the gettering area of the target and substrate is usually much larger than that of walls [3]. The reactive gas is mainly consumed by the metal part at target or substrate surfaces. Therefore, the consumption rates can be expressed as following $[3,6]$ :

$$
\begin{aligned}
& q_{\mathrm{t}}=F_{r} \alpha_{\mathrm{t}}\left(1-\theta_{\mathrm{t}}\right) A_{\mathrm{t}}, \\
& q_{\mathrm{s}}=F_{r} \alpha_{\mathrm{s}}\left(1-\theta_{\mathrm{s}}\right) A_{\mathrm{s}} .
\end{aligned}
$$

$F_{r}$ is the reactive gas flux and $\alpha_{\mathrm{t}}, \alpha_{\mathrm{s}}$ are the sticking coefficient, which could represent the probabilistic feature of the gettering effect. The outflow rate by the vacuum pump is followed by

$$
q_{\text {pump }}=\frac{p S}{k_{\mathrm{B}} T},
$$

where $p$ is the (partial) pressure of reactive gas and $S$ is the pumping speed (volume/time). The reactive gas flux can be found from kinetic theory by assuming a Maxwell distribution of molecular velocities. Skipping the tedious derivations [2], the result is

$$
F_{r}=\frac{p}{\sqrt{2 \pi m k_{\mathrm{B}} T}} .
$$

$F_{r}$ (molecules/unit area and time) is the neutral reactive 
molecules bombardment to all surfaces in the processing chamber, $m$ is the mass of reactive gas molecule. Substituting Eqs. (2)-(5) into Eq. (1), we can obtain the rate equation of reactive gas pressure $(p)$ in terms of input flow rate and fraction of compounds along with other parameters. In Berg's model, $q_{\text {in }}$ is the most important variable since a typical process is controlled by inflow rate through a mass flow controller (Fig. 1). However, Eq. (1) alone is not enough to draw a complete picture of the system dynamics, we need to further include the evolution of the fraction of compounds at the target and substrate surfaces.

Considering the mass balance at the target and substrate surfaces, the rate equations for compound formations can be found as

$$
\begin{aligned}
& \frac{\mathrm{d} \theta_{\mathrm{t}}}{\mathrm{d} t}=F_{r} \alpha_{\mathrm{t}}\left(1-\theta_{\mathrm{t}}\right) A_{\mathrm{t}} a_{\mathrm{t}}-\frac{J}{e} Y_{\mathrm{c}} \theta_{\mathrm{t}} A_{\mathrm{t}}, \\
& \frac{\mathrm{d} \theta_{\mathrm{s}}}{\mathrm{d} t}=F_{r} \alpha_{\mathrm{s}}\left(1-\theta_{\mathrm{s}}\right) A_{\mathrm{s}} a_{\mathrm{s}} \\
& \quad+\frac{J}{e} A_{\mathrm{t}}\left[Y_{\mathrm{c}} \theta_{\mathrm{t}}\left(1-\theta_{\mathrm{s}}\right)-Y_{\mathrm{m}} \theta_{\mathrm{s}}\left(1-\theta_{\mathrm{t}}\right)\right],
\end{aligned}
$$

where $\theta_{\mathrm{t}}$ is the compound fraction on target surface, $\theta_{\mathrm{s}}$ is the compound fraction on substrate surface, $a_{\mathrm{t}}, a_{\mathrm{c}}$ is the stoichiometric numbers for compound formation (number of compound molecules/reactive gas molecules) at the target and substrate, $Y_{\mathrm{c}}, Y_{\mathrm{m}}$ is the sputtering yield of compound (metal) at the target (atoms sputtered/incident ion) and $e$ is the unsigned charge of an electron, $A_{\mathrm{t}}, A_{\mathrm{c}}$ are target and substrate area, respectively. Physically, the first term in Eq. (6) represents the increase of compound molecules due to the reaction between reactive gas and metal part, whilst the second term is the reduction in compound sputtered by incident ions. Similar interpretation is applicable for Eq. (7) except that only the third term represents the reduction of compound fraction since the sputtered metal from target could replace some of the compounds at substrate.

\subsection{Effect of secondary electrons on target voltage}

The target voltage changes as a function of reactive gas partial pressure. This is due to the difference between the secondary emission coefficient of the metal and reaction product. When the target surface composition changes, the amount of secondary electrons emitted by ion bombardment of the target surface changes. This will, in turn, change the ratio between ion and electron currents. It is not possible to distinguish the electron and ion currents in the external electrical circuit. Therefore, a constant total current during processing does not necessarily imply that there is a constant ion current bombarding the target. If the secondary electron emission coefficient can be estimated for the different target surface conditions, this effect can be included in the modelling work. Some authors have done this $[8,9]$, and the results indicate that this effect does not change the general shape of the calculated curves. To some extent, however, it offers a possibility to include the plasma conditions into the calculations.
As target coverage by compound should strongly influence impedance of target-plasma system. We assume that target voltage can be expressed in terms of target compound fraction as

$$
U=U_{\text {me }}\left(1-\theta_{\mathrm{t}}\right)+U_{\mathrm{c}} \theta_{\mathrm{t}},
$$

where $U_{\mathrm{me}}$ and $U_{\mathrm{c}}$ are voltage limits measured far in compound and metallic mode, respectively.

\subsection{State space representation and equilibrium curves}

Defining a vector of variables containing the reactive gas partial pressure and compound fractions as

$$
X^{\mathrm{T}}=\left[p, \theta_{\mathrm{t}}, \theta_{\mathrm{c}}\right] \text {, }
$$

Eqs. (1), (6), (7) can be generally expressed as a set of first-order differential equations

$$
\frac{\mathrm{d} X}{\mathrm{~d} t}=F(X, u),
$$

where $F$ are the functions given in the right hand side of Eqs. (1), (6), (7) and $u=q_{\text {in }}$ is the input of this dynamical model. The output to be controlled is the target voltage $y=U$ given by Eq. (8) which can be written as

$$
y=G(X, u) .
$$

So the state representation of the nonlinear dynamical system is

$$
\left\{\begin{array}{l}
\frac{\mathrm{d} X}{\mathrm{~d} t}=F(X, u), \\
y=G(X, u) .
\end{array}\right.
$$

The equilibrium condition

$$
F(X, u)=0
$$

serves as an implicit definition of the function $X(u)$, i.e. the dependence of reactive gas partial pressure and compound fractions on the reactive gas flow in the equilibrium states. Figures 2 and 3 show the equilibrium curves

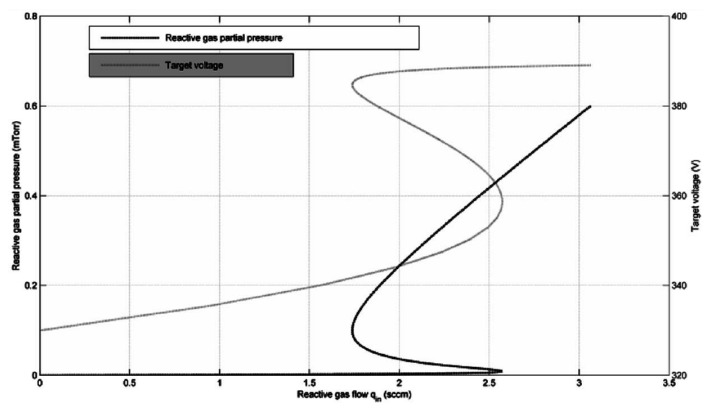

Fig. 2. Equilibrium curve of reactive gas partial pressure and target voltage vs. supply of the reactive gas.

of the dynamical system given by Eq. (13) calculated with the data for the case of sputtering from $\mathrm{Ti}$ target in $\mathrm{Ar} / \mathrm{O}_{2}$ plasma. The experimentally measured dependence of the $\mathrm{O}_{2}$ partial pressure upon the $\mathrm{O}_{2}$ inflow rate given in Ref. [10] is used for the calculation of target voltage. The experimental conditions were following: the power supplied to the target $800 \mathrm{~W}$, the partial pressure of Ar gas $0.9 \mathrm{~Pa}$, the target surface area $0.031 \mathrm{~m}^{2}$, the substrate surface area $0.20 \mathrm{~m}^{2}$, and the pumping speed 


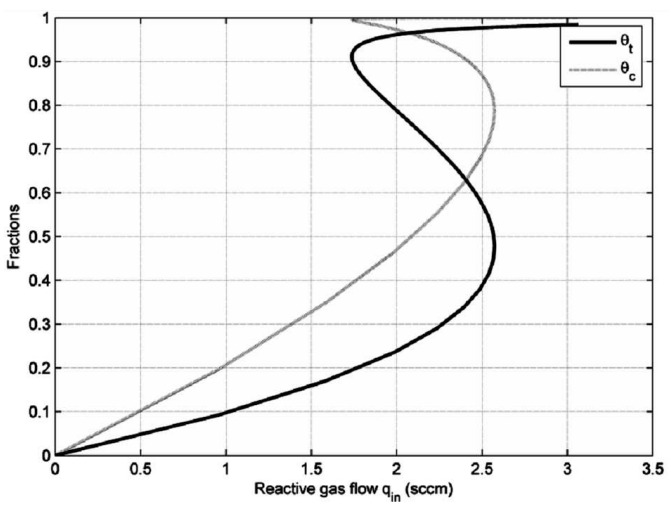

Fig. 3. Equilibrium curve of compound fractions $\theta_{\mathrm{t}}$ and $\theta_{\mathrm{c}}$ for the target and substrate, respectively, vs. supply of the reactive gas.

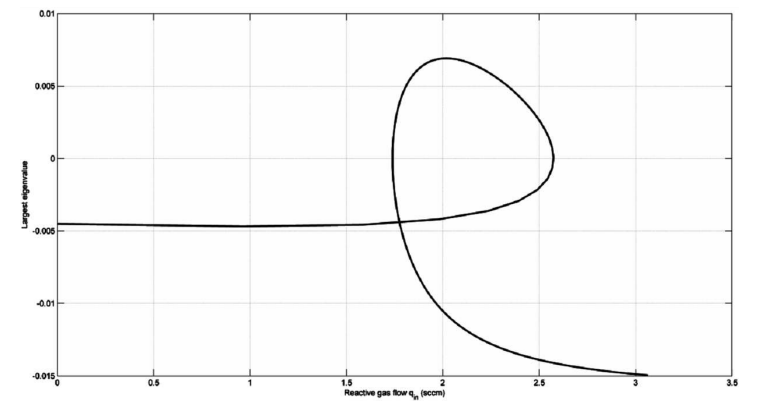

Fig. 4. The largest eigenvalue of matrix $A$ for various equilibrium states.

$0.050 \mathrm{~m}^{3} / \mathrm{s}$. There is a good agreement between experimental data and model as shown in Fig. 1.

\section{Results and discussion}

In order to discuss the controllability of the sputtering process, the result of linear control theory can be used. Given the nonlinear system (12) and an equilibrium point $X^{*}=\left[x_{1}^{*}, x_{2}^{*}, x_{3}^{*}\right]^{\mathrm{T}}$ obtained when $u=u^{*}$, a coordinate transformation as follows can be defined. Denote $\Delta X=X-X^{*}, \Delta u=u-u^{*}, \Delta y=y-y^{*}$. The linearization of $(12)$ at $X^{*}$ is given by

$$
\begin{gathered}
\Delta X=A \Delta X+B \Delta u, \\
\Delta y=C \Delta X+D \Delta u,
\end{gathered}
$$

where

$$
\begin{aligned}
& A=\left[\frac{\partial F}{\partial X}\right]_{X^{*}, u^{*}}, \quad B=\left[\frac{\partial F}{\partial u}\right]_{X^{*}, u^{*}}, \\
& C=\left[\frac{\partial G}{\partial X}\right]_{X^{*}, u^{*}}, \quad D=\left[\frac{\partial G}{\partial u}\right]_{X^{*}, u^{*}} .
\end{aligned}
$$

The linear system matrices $A, B, C, D$ can be analytically calculated in all points in the equilibrium curves shown in Figs. 2 and 3. The largest eigenvalue of matrix
$A$ for various equilibrium states is displayed in Fig. 4 . The negative value of the largest eigenvalue of matrix $A$ for low and high value of reactive gas flow rate, corresponds to the stable metallic and reactive modes. While the positive value at intermediate value of reactive gas flow rate corresponds to the unstable transition mode.

Controllability matrices $\left[B A B A^{2} B\right]$ are calculated in all points in the equilibrium curves and are full rank for all points in the equilibrium curves so the system is always controllable.

\section{Conclusion}

The controllability of reactive sputtering based upon Berg's model is characterized by dynamic solutions. For small variation away from the equilibrium state, a linear perturbation method is employed to form an eigenvalue problem where the instability is thus solely dictated by the positiveness of eigenvalues. It is shown that the eigenvalues are coherent to the existence of hysteresis loops, and the negative eigenvalues indicate that an unsteady system converges to steady state relatively fast at low inflow rates. In addition, the eigenvalue can be used to find the effect of parameters and ratios on stability, i.e. when the chemical reaction on substrate is moderate, a higher sputter yield of the compound leads to a more stable steady state at lower inflow rates. Unstable working points in the transition region can be stabilized via closed loop reactive gas control.

\section{Acknowledgments}

The authors would like to thank Dr. I. Othman (Director of the Atomic Energy Commission of Syria) for his advice and support for this work.

\section{References}

[1] R. Mientus, K. Ellmer, Surf. Coat. Technol. 116-119, 1093 (1999)

[2] I. Safi, Surf. Coat. Technol. 127, 203 (2000)

[3] S. Berg, T. Nyberg, H.-O. Blom, C. Nender, in: Handbook of Thin Film Process Technology, Eds. D.A. Glocker, S.I. Shah, Institute of Physics Publishing, Bristol, UK 1998, p. A5.3:1.

[4] J. Musil, P. Baroch, J. Vlcek, K.H. Nam, J.G. Han, Thin Solid Films 475, 208 (2005).

[5] Z.Y. Chen, A. Bogaerts, D. Depla, Nucl. Instrum. Methods Phys. Res. B 207, 415 (2003).

[6] S. Berg, T. Nyberg, Thin Solid Films 476, 215 (2005).

[7] D. Depla, S. Heirwegh, S. Mahieu, R. De Gryse, J. Phys. Appl. Phys. 40, 1957 (2007)

[8] A. Pflug, B. Szyszka, V. Sittinger, J. Niemann, in: Proc. 46th Annual SVC Technical Conf., Society of Vacuum Coaters, San Francisco, CA 2003, p. 241.

[9] Y. Matsuda, K. Otomo, H. Fujiyama, Thin Solid Films 390, 59 (2001)

[10] N. Martin, C. Rousselot, Surf. Coat. Technol. 110, 158 (1998) 